PROCEEDINGS OF THE

AMERICAN MATHEMATICAL SOCIETY

Volume 134, Number 10, October 2006, Pages 2873-2880

S 0002-9939(06)08327-4

Article electronically published on May 5, 2006

\title{
MARKOV TRACES ON CYCLOTOMIC TEMPERLEY-LIEB ALGEBRAS
}

\author{
HEBING RUI
}

(Communicated by John R. Stembridge)

\begin{abstract}
In this note, we use generalized Tchebychev polynomials to define a trace function which satisfies certain conditions. Such a trace will be called the Markov trace. In particular, we obtain formulae for the weights of the Markov trace. As a corollary, we get a combinatorial identity. This generalizes Jones's 1983 result on Temperley-Lieb algebras.
\end{abstract}

\section{INTRODUCTION}

The Temperley-Lieb algebras were first introduced in $[8$ in order to study the single bond transfer matrices for the Ising model. Later, they were independently found by Jones in [5] when he characterized the algebras arising from the tower construction of split semisimple algebras in the study of subfactors. In that case, the weights of a trace function defined on a Temperley-Lieb algebra play the key role. Such a trace is called the Markov trace.

In [6, $\mathrm{Xi}$ and the author introduced a class of finite-dimensional algebras called cyclotomic Temperley-Lieb algebras of type $G(m, 1, n)$. When $m=1$, it turns out to be the usual Temperley-Lieb algebra. Using generalized Tchebychev polynomials in [6], $\mathrm{Xi}, \mathrm{Yu}$ and the author gave a criterion for the semisimplicity of a cyclotomic Temperley-Lieb algebra in [7]. When $m=1$, such a result can be found in 2]. See also 9 .

Recall that a trace function is a central function, hence a linear combination of the irreducible characters, and the weights are the coefficients of the irreducible characters in such an expression. In [5], Jones shows that the weights of the Markov trace on the Temperley-Lieb algebra can be expressed via Tchebychev polynomials. It is natural to ask whether we can use our generalized Tchebychev polynomials to determine the Markov trace on the cyclotomic Temperley-Lieb algebra. In this note, we will answer this question. We hope that such a trace will be useful in the future.

We organize this note as follows. In section 2, we collect some of results on cyclotomic Temperley-Lieb algebras in [6] and [7]. We also state Theorem [2.9]

Received by the editors November 17, 2004 and, in revised form, March 12, 2005 and May 7, 2005 .

2000 Mathematics Subject Classification. Primary 16S99, 16K20.

The author was partially supported by NSFC no. 10331030 and JSPS. He wishes to thank the Research Institute for Mathematical Sciences, Kyoto University, for its hospitality during his visit.

(C)2006 American Mathematical Society Reverts to public domain 28 years from publication 
the main result of this note. We study the decompositions of induced modules in section 3. This will give the relationship between certain primitive idempotents. Via such relations, we can give a simple proof of Corollary 2.11, the existence part of the Theorem 2.9.

\section{Preliminaries AND THE MAIN THEOREM}

In this section, we recall the notion of a cyclotomic Temperley-Lieb algebra in [6] and state the main result in Theorem 2.9.

Definition $2.1\left([6)\right.$. Suppose $R$ is a field containing elements $\delta_{0}, \delta_{1}, \cdots, \delta_{m-1}$. The cyclotomic Temperley-Lieb algebra $T L_{m, n}\left(\delta_{0}, \cdots, \delta_{m-1}\right)$ is an associative algebra over $R$ generated by $e_{1}, e_{2}, \cdots, e_{n-1}$ and $t_{1}, t_{2}, \cdots, t_{n}$ subject to the following conditions:

(1) $e_{i} e_{j} e_{i}=e_{i}$, if $j=i \pm 1$,

(2) $e_{i} e_{j}=e_{j} e_{i}$, if $j \neq i \pm 1$,

(3) $t_{i}^{m}=1$, if $1 \leq i \leq n$,

(4) $t_{i} t_{j}=t_{j} t_{i}$, if $1 \leq i, j \leq n$,

(5) $e_{i} t_{i}^{k} e_{i}=\delta_{k} e_{i}$, if $0 \leq k \leq m-1$ and $1 \leq i \leq n-1$,

(6) $t_{i} t_{i+1} e_{i}=e_{i} t_{i} t_{i+1}=e_{i}$, if $1 \leq i \leq n-1$,

(7) $e_{i} t_{j}=t_{j} e_{i}$, if $j \neq i, i+1$.

Later on, we use $T L_{m, n}$ instead of $T L_{m, n}\left(\delta_{0}, \cdots, \delta_{m-1}\right)$. When $m=1, T L_{m, n}$ is the usual Temperley-Lieb algebra in [8].

2.2. Suppose $x_{1}, x_{2}, \cdots, x_{n}$ are indeterminates over $R$. Let $A_{n}=\left(a_{i j}\right)$ be the $n$-by- $n$ matrix with $a_{i i}=x_{i}, a_{i j}=1$ for $|i-j|=1$ and $a_{i j}=0$ in the remaining case. Following [6], we call

$$
P_{n}\left(x_{1}, x_{2}, \cdots, x_{n}\right):=\operatorname{det} A_{n}
$$

the $n$-th generalized Tchebychev polynomial in indeterminates $x_{1}, x_{2}, \cdots, x_{n}$.

2.3. Suppose $R$ contains $\xi$, a primitive $m$-th root of unity. For any $1 \leq i \leq m$, define $\bar{\delta}_{i} \in R$ by a system of linear functions

$$
\delta_{j}=\sum_{k=1}^{m} \bar{\delta}_{k} u_{k}^{j}, \text { for } 0 \leq j \leq m-1 .
$$

2.4. Fix two positive integers $m, n$. Following $\left[\underline{6}\right.$, write $\Lambda(m, n)=\left\{\left(i_{1}, i_{2}, \cdots, i_{n}\right) \mid\right.$ $\left.1 \leq i_{j} \leq m, 1 \leq j \leq n\right\}$.

The following result gives the necessary and sufficient condition for $T L_{m, n}$ to be semisimple. When $m=1$, such a result can be found in [2]. See also 9]. Note that the proof given in [7] is not valid for $m=1$ although Theorem 2.5 still holds true in this case.

Theorem 2.5 ([7, 4.7]). Suppose $m \geq 2$. Let $R$ be a splitting field of $x^{m}-1$. Then $T L_{m, n}$ is split semisimple if and only if

(a) $\operatorname{char} R \nmid m$.

(b) $\bar{\delta}_{i} \neq 0,1 \leq i \leq m$.

(c) Suppose $2 \leq l \leq n$. For any $\left(i_{1}, i_{2}, \cdots, i_{l}\right) \in \Lambda(m, l)$ with $m \mid\left(i_{j}+i_{j+1}\right), 1 \leq$ $j \leq l-1, P_{l}\left(\bar{\delta}_{i_{1}}, \bar{\delta}_{i_{2}}, \cdots, \bar{\delta}_{i_{l}}\right) \neq 0$. 
2.6. From here on, we assume $T L_{m, n}$ is split semisimple. By Theorem 2.5(b), there is at least one parameter which is not equal to zero. We will always assume $\delta_{0} \neq 0$ later on.

2.7. Let $\Lambda_{m, n}=\{(k, \boldsymbol{i}) \mid 0 \leq k \leq\lfloor n / 2\rfloor, \boldsymbol{i} \in \Lambda(m, n-2 k)\}$. It is proved in [6] that the simple $T L_{m, n}$-modules are indexed by $\Lambda_{m, n}$ if $T L_{m, n}$ is semisimple. In this case, let $p_{(k, i)}$ be the primitive idempotent with respect to the simple module $\Delta(k, \boldsymbol{i})$ defined in [6]. We remark that $\Delta(k, \boldsymbol{i})$ is a left $T L_{m, n}-$ module.

2.8. The cyclotomic Temperley-Lieb algebras are defined via labelled TemperleyLieb diagrams in [6]. Furthermore, $\mathrm{Xi}$ and the author prove that this definition is equivalent to Definition 2.1 in [6, 3.4]. Consequently, $T L_{m, n-1}$ can be considered as a subalgebra of $T L_{m, n}$ by adding a vertical arc to the right side of each labelled Temperley-Lieb diagram $D$ in $T L_{m, n-1}$. This embedding can be visualized as follows:

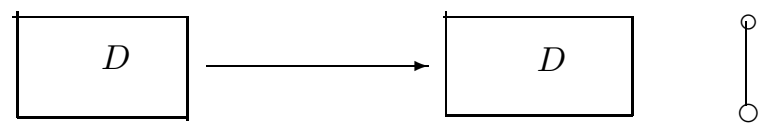

Let $\chi_{(k, \boldsymbol{i})}$ be the irreducible character with respect to $\Delta(k, \boldsymbol{i})$. Recall that a linear function on an $R$-algebra $A$ is called a trace function if $f(x y)=f(y x)$ for all $x, y \in A$.

Theorem 2.9. Suppose $\delta_{0} \neq 0$ and $T L_{m, n}$ are split semisimple over the field $R$ for all $n$. Then there exists a unique $R$-linear function $\operatorname{tr}: \bigcup_{n=1}^{\infty} T L_{m, n} \rightarrow R$ satisfying the conditions (a) to (e) below. We use $\operatorname{tr} \downarrow_{T L_{m, n}}$ to denote the restriction of tr to $T L_{m, n}$.

(a) $\operatorname{tr} \downarrow_{T L_{m, n}}(w)=\operatorname{tr} \downarrow_{T L_{m, n-1}}(w)$ if $w \in T L_{m, n-1}$,

(b) $\operatorname{tr}(1)=1$,

(c) $\operatorname{tr}\left(w e_{n}\right)=\delta_{0}^{-1} \operatorname{tr}(w)$, if $w \in T L_{m, n}$,

(d) $\operatorname{tr}\left(w t_{n+1}^{k}\right)=\delta_{k} \delta_{0}^{-1} \operatorname{tr}(w)$ if $w \in T L_{m, n}$,

(e) $\operatorname{tr} \downarrow_{T L_{m, n}}$ is non-degenerate for all $n$.

Proof. By [6, 3.4, 2.4, 2.2], we have, as a free $R$-module,

$$
T L_{m, n}=\bigoplus_{j=0}^{m-1} T L_{m, n-1} t_{n}^{j} \bigoplus T L_{m, n-1} e_{n-1} T L_{m, n-1}
$$

This implies that a trace function on $T L_{m, n}$ satisfying Theorem $2.9(\mathrm{~b})-(\mathrm{d})$ is unique. Condition (a) in Theorem 2.9 shows that $t r$ is well defined on $\bigcup_{n=1}^{\infty} T L_{m, n}$. The existence part of the theorem follows from Corollary 2.11, which will be proved in section 3 .

Remark 2.10. (a) The trace in Theorem 2.9 will be called the Markov trace on $\bigcup_{n=1}^{\infty} T L_{m, n}$. Consequently, $t r \downarrow_{T L_{m, n}}$ is the Markov trace function on $T L_{m, n}$. By Corollary 2.11, we know the weights of Markov trace with respect to all $(k, \boldsymbol{i}) \in$ $\Lambda_{m, n}$.

(b) The trace function studied in Theorem 2.9 and Corollary 2.11 is a generalization of Jones's trace function in [5]. Also the Jones' trace is descent to $T L_{1, n}$ of Ocneanu's trace on the type $A$ Hecke algebra. However, it is unclear whether there 
is a similar relationship between the cyclotomic Temperley-Lieb algebras and ArikiKoike algebras in [1. The reason is that we do not know whether the cyclotomic Temperley-Lieb algebra is a quotient of the Ariki-Koike algebra.

(c) There is another version of type $B$ Temperley-Lieb algebras called blob algebras by mathematical physicists, which has been much studied in [4, §5]. However, a blob algebra is not the same as our $T L_{2, n}$. One can compare our generators $t_{i}$ with $c_{0}$ in [4,5.3].

(d) We have that $T L_{m, n}$ are split semisimple for all $n$ if and only if Theorem 2.5(a) holds together with $P_{l}\left(\bar{\delta}_{i_{1}}, \bar{\delta}_{i_{2}}, \cdots, \bar{\delta}_{i_{l}}\right) \neq 0$ for all positive integers $l$ and $\left(i_{1}, i_{2}, \cdots, i_{l}\right) \in \Lambda(m, l)$. Also, we have $T L_{m, k}$ is split semisimple if $T L_{m, n}$ is semisimple and $k \leq n$. This follows directly from Theorem 2.5 .

Corollary 2.11. Suppose $\delta_{0} \neq 0$ and $T L_{m, n}$ are split semisimple over the field $R$ for all $n$. For any $(k, \boldsymbol{i}) \in \Lambda_{m, n-2 k}$, let $\boldsymbol{i}$ be divided into $\left(i_{1,1}, \cdots, i_{1, j_{1}}, i_{2,1}, \cdots, i_{2, j_{2}}\right.$, $\left.\cdots, i_{r, j_{r}}\right)$ such that

(1) $\sum_{l=1}^{r} j_{l}=n-2 k$,

(2) $m \mid i_{p, q}+i_{p, q+1}$ for $1 \leq p<r$ and $1 \leq q<j_{p}$,

(3) $m \nmid i_{p, j_{p}}+i_{p+1,1}$ for $1 \leq p<r$.

For any $m, n$, let $\tau_{m, n}: T L_{m, n} \rightarrow R$ be the trace function defined by setting

$$
\tau_{m, n}=\sum_{(k, i) \in \Lambda_{m, n}} \delta_{0}^{-n} \prod_{l=1}^{r} P_{j_{l}}\left(\bar{\delta}_{i_{l, 1}}, \bar{\delta}_{i_{l, 2}}, \cdots, \bar{\delta}_{i_{l, j_{l}}}\right) \chi_{(k, \boldsymbol{i})} .
$$

Then

(1) $\tau_{m, n+1}(w)=\tau_{m, n}(w)$, if $w \in T L_{m, n}$.

(2) $\tau_{m, n}(1)=1$

(3) $\tau_{m, n+1}\left(w e_{n}\right)=\delta_{0}^{-1} \tau_{m, n}(w)$, if $w \in T L_{m, n}$.

(4) $\tau_{m, n+1}\left(w t_{n+1}^{k}\right)=\delta_{k} \delta_{0}^{-1} \tau_{m, n}(w)$, if $w \in T L_{m, n}$.

(5) $\tau_{m, n}$ is non-degenerate.

Consequently, $\tau_{m, n}=\operatorname{tr} \downarrow_{T L_{m, n}}$, where $\operatorname{tr}$ is the trace function in Theorem 2.9.

Proof. The last assertion follows from the fact that the trace function on $T L_{m, n}$ satisfying conditions (2)-(4) is unique. We will prove the first assertion in section 3.

When $m=1$, the sequence $(1,1, \cdots, 1)$ can be divided into only one part and a generalized Tchebychev polynomial is the usual Tchebychev polynomial. In this case, Theorem 2.9 was proved by Jones in [5, §5]. The following result follows from Corollary 2.11 and [6, 5.5], immediately.

Corollary 2.12. Let $T L_{m, n}$ be split semisimple over a field $R$ and let $\delta_{0} \neq 0$ in R. Then

$$
1=\sum_{(k, i) \in \Lambda_{m, n}} \delta_{0}^{-n} m^{k}\left[\left(\begin{array}{c}
n \\
k
\end{array}\right)-\left(\begin{array}{c}
n \\
k-1
\end{array}\right)\right] \prod_{l=1}^{r} P_{j_{l}}\left(\bar{\delta}_{i_{l, 1}}, \bar{\delta}_{i_{l, 2}}, \cdots, \bar{\delta}_{i_{l, j_{l}}}\right) .
$$

\section{Proof of Corollary 2.11}

In this section, we assume $\delta_{0} \neq 0$ and $T L_{m, n}$ are split semisimple over a field $R$ for all $n$. One can translate the latter assumption into a condition on the parameters $\delta_{i}$ explicitly by Theorem 2.5. 
Definition 3.1. Let $A_{m, n}$ be the subalgebra of $T L_{m, n}$ generated by $e_{1}, \cdots, e_{n-2}$, $t_{1}, t_{2}, \cdots, t_{n}$. Then $A_{m, n}=T L_{m, n-1} \otimes_{R} G_{n}$, where $G_{n}$ is the group algebra of the cyclic group $\left\langle t_{n}\right\rangle$. Since $T L_{m, n}$ is split semisimple for any $n$, so is $A_{m, n}$.

3.2. By a general result on the tensor product of two algebras, we see that the simple $A_{m, n}$-modules are of forms $\Delta(k, \boldsymbol{i}) \otimes \Delta(j)$, where $(k, \boldsymbol{i}) \in \Lambda_{m, n-1}$ and $\Delta(i)$ is the simple $G_{n}$-module on which $t_{n}$ acts as $u_{i}=\xi^{i}$.

Lemma 3.3. Suppose $(k, i) \in \Lambda_{m, n}$. Then

(a) $\Delta(k, i) \downarrow \cong \bigoplus_{l=1}^{m} \Delta(k-1, i \cup l) \otimes \Delta(m-l) \oplus \Delta\left(k, i \backslash\left\{i_{n-2 k}\right\}\right) \otimes \Delta\left(i_{n-2 k}\right)$. Here $\Delta(k, \boldsymbol{i}) \downarrow$ denotes the restriction of the $T L_{m, n}-\operatorname{module} \Delta(k, \boldsymbol{i})$ to $A_{m, n}$.

(b) $\Delta(k, \boldsymbol{i}) \otimes \Delta(j) \uparrow \cong \begin{cases}\Delta(k, \boldsymbol{i} \cup j), & \text { if } j \neq m-i_{n-2 k}, \\ \Delta(k, \boldsymbol{i} \cup j) \oplus \Delta\left(k+1, i \backslash i_{n-2 k}\right), & \text { if } j=m-i_{n-2 k},\end{cases}$ where $\Delta(k, i) \otimes \Delta(j) \uparrow$ denotes the induction of the $A_{m, n+1}-$ module $\Delta(k, i) \otimes \Delta(j)$ to $T L_{m, n+1}$.

Proof. It is not difficult to see that (b) follows from (a) and Frobenius reciprocity. We can prove (a) by the similar arguments in the proof of [6, 7.1]. We just point out the difference and leave the details to the reader.

The $T L_{m, n-1}$-submodule of $\Delta(k, i)$ given in the proof of [6, 7.1], which is isomorphic to $\Delta\left(k, \boldsymbol{i} \backslash\left\{i_{n-2 k}\right\}\right)$, is isomorphic to $\Delta\left(k, \boldsymbol{i} \backslash\left\{i_{n-2 k}\right\}\right) \otimes \Delta\left(i_{n-2 k}\right)$ if the submodule is considered as an $A_{m, n}$-module. This can be verified easily by considering the action of $t_{n}$ on it.

The $T L_{m, n-1}$-homomorphism $\beta$ in the proof of [6, 7.1] is an $A_{m, n}$-homomorphism if we let $t_{n}$ act the same as $t_{j}^{-1}$ on $\beta(D)$, where $\{j, n\}$ is the horizontal arc at the top row of $D$. The trick is relation (6) in Definition 2.1, which is equivalent to saying a dot will be replaced by $m-1$ dots if it moves from one endpoint of a horizontal arc to another (see [6, §3] for details). This implies that the short exact sequence given in [7, (2.3)] is an $A_{m, n}$-module short exact sequence and $t_{n}$ acts on $\Delta(k-1, i \cup j)$ as the scalar $u_{m-j}$. In other words, there is an $A_{m, n^{-}}$ module isomorphism $\Delta(k-1, i \cup j) \cong \Delta(k-1, \boldsymbol{i} \cup j) \otimes_{R} \Delta(m-j)$. Since $A_{m, n}$ is semisimple, the short exact sequence given in [7, (2.3)] is split. This completes the proof of (a).

Lemma 3.4. Let $B_{m, n+2}$ be the subalgebra of $T L_{m, n+2}$ generated by $T L_{m, n}$ and $e_{n+1}$. Let $\tilde{\Delta}(1,0)$ be the simple $\left\langle e_{n+1}\right\rangle$-module on which $e_{n+1}$ acts non-trivially, where $\left\langle e_{n+1}\right\rangle$ is the subalgebra generated by $e_{n+1}$. For any $(k, \boldsymbol{i}) \in \Lambda_{m, n}, \Delta(k, \boldsymbol{i}) \otimes_{R}$ $\tilde{\Delta}(1,0) \uparrow \cong \Delta(k+1, \boldsymbol{i})$, where $\Delta(k, \boldsymbol{i}) \otimes_{R} \tilde{\Delta}(1,0) \uparrow$ is the induction of a $B_{m, n+2}-$ module $\Delta(k, i) \otimes_{R} \tilde{\Delta}(1,0)$ to $T L_{m, n+2}$.

Proof. We first point out that $B_{m, n+2}$ is split semisimple since $T L_{m, n}$ is split semisimple and $\delta_{0} \neq 0$. By [6, 5.4] and the definition of a cell module in [3], the induced module $\Delta(k, \boldsymbol{i}) \otimes_{R} \tilde{\Delta}(1,0) \uparrow$ contains a simple submodule which is isomorphic to the $T L_{m, n+2}-$ module $\Delta(k+1, i)$. Recall that $p_{(k, i)}$ and $\delta_{0}^{-1} e_{n+1}$ are primitive idempotents with respect to the simple modules $\Delta(k, i)$ and $\tilde{\Delta}(1,0)$. Since $p_{(k, \boldsymbol{i})} e_{n+1}=e_{n+1} p_{(k, \boldsymbol{i})}, \delta_{0}^{-1} p_{(k, \boldsymbol{i})} e_{n+1}$ is an idempotent. Noting that $\Delta(k, \boldsymbol{i})$ is irreducible, we have

$$
p_{(k, i)} T L_{m, n} p_{(k, i)} \cong R
$$


It follows from [7, 3.1] that $e_{n+1} T L_{m, n+2} e_{n+1}=e_{n+1} T L_{m, n}$. Consequently,

$$
\begin{aligned}
p_{(k, \boldsymbol{i})} e_{n+1} T L_{m, n+2} e_{n+1} p_{(k, \boldsymbol{i})} & =p_{(k, \boldsymbol{i})} e_{n+1} T L_{m, n} p_{(k, \boldsymbol{i})} \\
& =e_{n+1} p_{(k, \boldsymbol{i})} T L_{m, n} p_{(k, \boldsymbol{i})} \cong R \text { by (3.1). }
\end{aligned}
$$

Therefore, $\Delta(k, \boldsymbol{i}) \otimes_{R} \tilde{\Delta}(1,0) \uparrow$ is irreducible, which implies the desired isomorphism.

By [7, 2.6] and Frobenius reciprocity, we have the following result.

Lemma 3.5. For any $(k, \boldsymbol{i}) \in \Lambda_{m, n}$, there is a $T L_{m, n+1}-$ module isomorphism

$$
\Delta(k, \boldsymbol{i}) \uparrow \cong \bigoplus_{j=1}^{m} \Delta(k, \boldsymbol{i} \cup\{j\}) \oplus \Delta\left(k+1, \boldsymbol{i} \backslash\left\{i_{n-2 k}\right\}\right),
$$

where $\Delta(k, \boldsymbol{i}) \uparrow$ denotes the induction of the $T L_{m, n}-$ module $\Delta(k, \boldsymbol{i})$ to $T L_{m, n+1}$.

3.6. Proof of Corollary 2.11. We verify $\tau_{m, n+1}(w)=\tau_{m, n}(w)$ for all $w \in T L_{m, n}$ at first. Without loss of generality, we can assume $w$ is a primitive idempotent, say $p_{(k, \boldsymbol{i})}$. By Lemma 3.5. $p_{(k, i)}=\sum_{j=1}^{m} p_{(k, i \cup\{j\})}+p_{\left(k+1, i \backslash\left\{i_{n-2 k}\right\}\right)}$. Since $m>1$, for $1 \leq j \leq m$, there is a unique number, say $j_{0}$, such that $m \mid i_{n-2 k}+j_{0}$. It follows from (2.4) that

- $\tau_{m, n+1}\left(p_{(k, i \cup\{j\})}\right)=\bar{\delta}_{j} \delta_{0}^{-n-1} \prod_{l=1}^{r} P_{j_{l}}\left(\bar{\delta}_{i_{l, 1}}, \bar{\delta}_{i_{l, 2}}, \cdots, \bar{\delta}_{i_{l, j_{l}}}\right), \quad j \neq j_{0}$,

- $\tau_{m, n+1}\left(p_{\left(k, i \cup\left\{j_{0}\right\}\right)}\right)=\delta_{0}^{-n-1} P_{j_{r}+1}\left(\bar{\delta}_{i_{r, 1}}, \bar{\delta}_{i_{r, 2}}, \cdots, \bar{\delta}_{i_{r, j_{r}}}, \bar{\delta}_{j_{0}}\right)$

$$
\times \prod_{l=1}^{r-1} P_{j_{l}}\left(\bar{\delta}_{i_{l, 1}}, \bar{\delta}_{i_{l, 2}}, \cdots, \bar{\delta}_{i_{l, j_{l}}}\right),
$$

- $\tau_{m, n+1}\left(p_{\left(k+1, i \backslash\left\{i_{n-2 k}\right\}\right)}\right)=\delta_{0}^{-n-1} P_{j_{r}-1}\left(\bar{\delta}_{i_{r, 1}}, \bar{\delta}_{i_{r, 2}}, \cdots, \bar{\delta}_{i_{r, j_{r}-1}}\right)$

$$
\times \prod_{l=1}^{r-1} P_{j_{l}}\left(\bar{\delta}_{i_{l, 1}}, \bar{\delta}_{i_{l, 2}}, \cdots, \bar{\delta}_{i_{l, j_{l}}}\right) .
$$

By the definition of generalized Tchebychev polynomials in (2.1), we have

- $P_{n}\left(x_{1}, x_{2}, \cdots, x_{n}\right)=x_{n} P_{n-1}\left(x_{1}, x_{2}, \cdots, x_{n-1}\right)-P_{n-2}\left(x_{1}, x_{2}, \cdots, x_{n-2}\right)$.

Consequently,

$$
\begin{aligned}
\tau_{m, n+1}\left(p_{(k, \boldsymbol{i})}\right) & =\sum_{j=1}^{m} \tau_{m, n+1}\left(p_{(k, i \cup\{j\})}\right)+\tau_{m, n+1}\left(p_{\left(k+1, i \backslash\left\{i_{n-2 k}\right\}\right)}\right) \\
& =\delta_{0}^{-n-1} \sum_{j \neq j_{0}}^{m} \bar{\delta}_{j} \prod_{l=1}^{r} P_{j_{l}}\left(\bar{\delta}_{i_{l, 1}}, \bar{\delta}_{i_{l, 2}}, \cdots, \bar{\delta}_{i_{l, j_{l}}}\right) \\
& +\delta_{0}^{-n-1} \bar{\delta}_{j_{0}} \prod_{l=1}^{r} P_{j_{l}}\left(\bar{\delta}_{i_{l, 1}}, \bar{\delta}_{i_{l, 2}}, \cdots, \bar{\delta}_{i_{l, j_{l}}}\right) \\
& =\delta_{0}^{-n} \prod_{l=1}^{r} P_{j_{l}}\left(\bar{\delta}_{i_{l, 1}}, \bar{\delta}_{i_{l, 2}}, \cdots, \bar{\delta}_{i_{l, j_{l}}}\right) \text { by (2.2) } \\
& =\tau_{m, n}\left(p_{(k, i)}\right) .
\end{aligned}
$$


This shows (1). In particular, $\tau_{m, n}(1)=\tau_{m, 1}(1)$. Since $1=\sum_{j=1}^{m} p_{j}$, where $p_{j}$ is the primitive idempotent with respect to the simple module $\Delta(j)$, we have $\tau_{m, 1}(1)=\sum_{j=1}^{m} \tau_{m, 1}\left(p_{j}\right)=\delta_{0}^{-1} \sum_{j=1}^{m} \bar{\delta}_{j}=1$ by (2.2). Now, we prove (4). By Lemma 3.3(b),

$$
p_{(k, \boldsymbol{i})} p_{j}= \begin{cases}p_{(k, \boldsymbol{i} \cup\{j\})}, & \text { if } m \nmid i_{n-2 k}+j, \\ p_{(k, \boldsymbol{i} \cup\{j\})}+p_{\left(k+1, i \backslash\left\{i_{n-2 k}\right\}\right)}, & \text { otherwise. }\end{cases}
$$

Using (3.2), (2.2) and the recurrence formula for generalized Tchebychev polynomials, we have $\tau_{m, n+1}\left(p_{(k, i)} t_{n+1}^{l}\right)=\delta_{l} \delta_{0}^{-1} \tau_{m, n}\left(p_{(k, i)}\right)$, proving (4). In order to prove $(3)$, we need to consider three cases as follows.

Case 1: $w \in T L_{m, n-1}$. Without loss of generality, we can assume $w=p_{(k, i)}$, $(k, \boldsymbol{i}) \in \Lambda_{m, n-1}$. By Lemma 3.5, $\delta_{0}^{-1} p_{(k, \boldsymbol{i})} e_{n}=p_{(k+1, i)}$ for any $(k, \boldsymbol{i}) \in \Lambda_{m, n-1}$. So,

$$
\begin{aligned}
\tau_{m, n+1}\left(p_{(k, i)} e_{n}\right) & =\delta_{0} \tau_{m, n+1}\left(p_{(k+1, i)}\right)=\delta_{0} \delta_{0}^{-n-1} \prod_{l=1}^{r} P_{j_{l}}\left(\bar{\delta}_{i_{l, 1}}, \bar{\delta}_{i_{l, 2}}, \cdots, \bar{\delta}_{i_{l, j_{l}}}\right) \\
& =\delta_{0}^{-1} \tau_{m, n-1}\left(p_{(k, i)}\right) .
\end{aligned}
$$

Case 2: $w \in T L_{m, n-1}$. We have

$$
\begin{aligned}
\tau_{m, n+1}\left(w t_{n}^{l} e_{n}\right) & =\delta_{0}^{-1} \tau_{m, n+1}\left(w t_{n}^{l} e_{n}^{2}\right)=\delta_{0}^{-1} \delta_{l} \tau_{m, n+1}\left(w e_{n}\right) \\
& =\delta_{0}^{-2} \delta_{l} \tau_{m, n-1}(w)=\delta_{0}^{-1} \tau_{m, n}\left(w t_{n}^{l}\right) .
\end{aligned}
$$

The second equality follows from the relations (2), (5) and (7) in Definition 2.1. The third equality follows from the result in Case 1. Finally, the last equality follows from (4), which has already been proved.

Case 3: $w=w_{1} e_{n-1} w_{2}, w_{i} \in T L_{m, n-1}$. We have

$$
\begin{aligned}
\tau_{m, n+1}\left(w e_{n}\right) & =\tau_{m, n+1}\left(w_{1} e_{n-1} w_{2} e_{n}\right)=\delta_{0}^{-1} \tau_{m, n+1}\left(w_{1} e_{n-1} w_{2} e_{n}^{2}\right) \\
& =\delta_{0}^{-1} \tau_{m, n+1}\left(w_{1} e_{n} e_{n-1} e_{n} w_{2}\right) \\
& =\delta_{0}^{-1} \tau_{m, n+1}\left(w_{1} w_{2} e_{n}\right)=\delta_{0}^{-2} \tau_{m, n-1}\left(w_{1} w_{2}\right) \text { by Case } 1 .
\end{aligned}
$$

By induction assumption on $n, \tau_{m, n}\left(w_{1} e_{n-1} w_{2}\right)=\delta_{0}^{-1} \tau_{m, n-1}\left(w_{1} w_{2}\right)$. Therefore, $\tau_{m, n+1}\left(w e_{n}\right)=\delta_{0}^{-1} \tau_{m, n}(w)$. By the results in Case 1-Case 3 and (2.3), we see that (3) holds true. Finally, we have (5) by Theorem 2.5 since $T L_{m, n}$ is split semisimple.

\section{REFERENCES}

[1] S. Ariki and K. Koike, A Hecke algebra of $\mathbb{Z}_{r} \prec \mathfrak{S}_{n}$ and the construction of its irreducible representations, Adv. Math. 106 (1994), 216-243. MR.1279219 (95h:20006)

[2] F. Goodman, P. de la Harpe and V. F. R. Jones, Coxeter graphs and towers of algebras, Mathematical Sciences Research Institute Publications, 14 Springer-Verlag, New York, 1989. MR0999799 (91c:46082)

[3] J. Graham and G. Lehrer, Cellular algebras, Invent. Math. 123 (1996), 1-34. MR.1376244 (97h:20016)

[4] J. Graham and G. Lehrer, Diagram algebras, Hecke algebras and decomposition numbers at the root of unity, Ann. Sci. École. Norm. Sup. (4) 36 (2003), no. 4, 479-524. MR2013924 (2004k:20007)

[5] V.F.R. Jones, Index for Subfactors, Invent. Math. 72 (1983), 1-25. MR0696688 (84d:46097) 
[6] H. Rui and C. Xi, The representation theory of the cyclotomic Temperley-Lieb algebras, Comment. Math. Helv. 79 (2004), 427-450. MR2059440 (2005b:16027)

[7] H. Rui, C. Xi, and W. Yu, On the semisimplicity of cyclotomic Temperley-Lieb algebras, Michigan Math. Journal 53 (2005), no. 1, 83-96. MR2125535

[8] H.N.V. Temperley and E.H. Lieb, Relations between percolation and colouring problems and other graph theoretical problems associated with regular planar lattices: some exact results for the percolation problem, Proc. Roy. Soc. London (Ser. A) 322 (1971), 251-273. MR0498284 (58:16425)

[9] B. Westbury, The representation theory of Temperley-Lieb algebras, Math. Z. 219 (1995), 539-565. MR1343661 (96h:20029)

Department of Mathematics, East China Normal University, Shanghai, 200062, PeoPle's RePUBlic OF ChinA

E-mail address: hbrui@math.ecnu.edu.cn 